\title{
Blood Management in Total Knee Arthroplasty: Updates and Debates
}

\author{
Kang-Il Kim, MD, PhD \\ Department of Orthopaedic Surgery, Kyung Hee University School of Medicine, Seoul, Korea
}

Hemodynamic change related to TKA has been considered a major issue since it can result in hematoma, blood transfusion, periposthetic joint infection, delayed rehabilitation, and prolonged hospitalization as well as serious hemodynamic complications such as deep vein thrombosis and pulmonary embolism ${ }^{1,2)}$. The theme of this issue of the Knee Surgery \& Related Research is blood management in TKA. The current issue contains four articles including one review article written by experts in blood management. Following the article that nicely summarizes recent updates, this issue presents three articles focusing on recently emerging but relatively controversial topics such as tranexamic acid (TXA) administration and various methods for postoperative drainage in order to generate the interest of the readers of the journal and the need for further related researches.

It has been reported that the amount of blood loss after TKA ranges from 1,400 to $1,800 \mathrm{~mL}$, and accordingly the frequency of blood transfusion can be as high as $40 \%{ }^{3,4)}$. Therefore, various blood conservation strategies for reducing perioperative bleeding and transfusion have been attempted including hypotensive anesthesia, autologous blood transfusion, intraoperative cell saver, plugging of the femoral canal, cementing, drain clamping, navigation, minimally invasive surgery, pneumatic tourniquet application, and the use of TXA ${ }^{3,5,6)}$. However, it is almost unfeasible to select one or two best approaches. Thus, the recent trend is

Correspondence to: Kang-Il Kim, MD, PhD

Department of Orthopaedic Surgery, Center for Joint Diseases, Kyung

Hee University Hospital at Gangdong, 892 Dongnam-ro, Gangdong-gu, Seoul 05278, Korea

Tel: +82-2-440-6151, Fax: +82-2-440-7468

E-mail:kneedr@hotmail.com

This is an Open Access article distributed under the terms of the Creative Commons Attribution Non-Commercial License (http://creativecommons.org/licenses/by-nc/4.0/) which permits unrestricted non-commercial use, distribution, and reproduction in any medium, provided the original work is properly cited. towards multimodal approaches efficiently incorporating various effective methods. The article by Liu et al. reviews the literature related to blood management strategies in the preoperative, intraoperative, and postoperative periods in TKA. The authors highlight the importance of preoperative hemoglobin optimization, minimal blood loss, and evidence-based guidelines. Moreover, it addresses an individualized, patient-specific multimodal approach and argues the "zero" allogenic postoperative transfusion rate should be the aim and an achievable goal of successful TKA procedures.

TXA, an anti-fibrinolytic agent, is a plasminogen inhibitor that is widely recognized as easy to administer and efficient for reduction of blood loss in major surgical procedures including joint replacement surgeries. The efficacy and safety of TXA in reducing blood loss have been demonstrated in numerous level 1 studies and high quality meta-analyses ${ }^{4,7)}$. On the other hand, since TXA affects the fibrinolytic system, it carries the risk of thromboembolic events ${ }^{8,9)}$. Postoperative venous thromboembolism is a potentially devastating complication of arthroplasties. Thus, due to the concern that the use of TXA may increase the risk of this serious complication, the efficacious agent for lowering postoperative bleeding and transfusion has been questioned or even avoided until recently in arthroplasty patients since its introduction in the 1960s. However, a number of recent prospective studies have reported that the incidence of deep vein thrombosis and pulmonary embolism did not increase in the TXA-administered group compared to the control group ${ }^{7}$. Seol et al. investigated the effect of TXA to reduce blood loss and postoperative transfusion following TKA. The prospective study compared an intravenous TXA group and a placebo group. They concluded that TXA can decrease total blood loss and postoperative transfusion following TKA.

In addition, pneumatic tourniquets are utilized by most TKA surgeon due to the advantages of preventing intraoperative www.jksrr.org 
bleeding and facilitating the surgical process for clear operative field and cementing; however, it also raises the concern of delayed bleeding and deep vein thrombosis ${ }^{10}$. Drains have been used by most surgeons to prevent postoperative hematomas and infections. It appears that the number of drains inserted and size of drain are determined by the surgeon's discretion. Lately, a growing body of studies have shown that postoperative bleeding and transfusion could be reduced significantly without the use of drainage by creating a tamponade effect ${ }^{11}$. Theoretically, without the use of drainage, blood accumulation from the cutting surface and medullary cavity should result in hematoma formation, which eventually causes surgical site pain, development of ecchymoses, and disruption of rehabilitation involving flexion exercises. However, it has been controversially highlighted in some studies that no use of a drain does not result in differences or produces even better results ${ }^{11,12)}$. In the meantime, some studies have shown that neutral drainage or drain clamping could reduce postoperative total blood loss by creating a tamponade effect compared to continuous negative pressure drainage ${ }^{11,13)}$. Following two articles published in the current theme address this topic. Kim et al. compared blood loss between neutral drainage with TXA and negative pressure drainage without TXA. Although it seems difficult to draw a definite conclusion due to the involvement of multiple variables (drainage, TXA, unilateral/bilateral TKA), they suggested that the use of intravenous TXA with 3-hour neutral drainage after TKA could be helpful to reduce total blood loss specifically in bilateral TKA. Lastly, the effect of closed suction drain on blood loss and transfusion is discussed by Jhurani et al. This prospective study, based on the comparison of no-drain group and the drain group, indicates no-drainage provides no hemodynamic advantage over drainage in terms of blood loss, transfusion, and complications in simultaneous bilateral TKAs.

The current issue has mainly focused on the theme of blood management to discuss its importance and various perioperative blood conservation methods utilized in TKA. Many researchers have tried to reduce blood loss in this common procedure with various methods in pre-, intra-, and postoperative periods. Experts have summarized and presented contemporary information and debate on blood conservation in TKA. Further researches on blood management are required for the purpose of minimizing complications and optimizing clinical results.

\section{References}

1. Levine BR, Haughom B, Strong B, Hellman M, Frank RM. Blood management strategies for total knee arthroplasty. J Am Acad Orthop Surg. 2014;22:361-71.
2. Tartiere J, Berthelin C, Jehan C, Trahay A, Samii K, Quesnel J. Hemodynamic changes during total knee replacement surgery with total condylar prosthesis. Anesthesiology. 1987;67:838-41.

3. Cankaya D, Della Valle CJ. Blood loss and transfusion rates in the revision of unicompartmental knee arthroplasty to total knee arthroplasty are similar to those of primary total knee arthroplasty but are lower compared with the revision total knee arthroplasty. J Arthroplasty. 2016;31:339-41.

4. Yang ZG, Chen WP, Wu LD. Effectiveness and safety of tranexamic acid in reducing blood loss in total knee arthroplasty: a meta-analysis. J Bone Joint Surg Am. 2012;94:1153-9.

5. Lee GC, Hawes T, Cushner FD, Scott WN. Current trends in blood conservation in total knee arthroplasty. Clin Orthop Relat Res. 2005;440:170-4.

6. Gannon DM, Lombardi AV Jr, Mallory TH, Vaughn BK, Finney CR, Niemcryk S. An evaluation of the efficacy of postoperative blood salvage after total joint arthroplasty: a prospective randomized trial. J Arthroplasty. 1991;6:109-14.

7. Lee SH, Cho KY, Khurana S, Kim KI. Less blood loss under concomitant administration of tranexamic acid and indirect factor Xa inhibitor following total knee arthroplasty: a prospective randomized controlled trial. Knee Surg Sports Traumatol Arthrosc. 2013;21:2611-7.

8. Ker K, Edwards P, Perel P, Shakur H, Roberts I. Effect of tranexamic acid on surgical bleeding: systematic review and cumulative meta-analysis. BMJ. 2012;344:e3054.

9. Pinsornsak P, Rojanavijitkul S, Chumchuen S. Peri-articular tranexamic acid injection in total knee arthroplasty: a randomized controlled trial. BMC Musculoskelet Disord. 2016;17:313.

10. Abdel-Salam A, Eyres KS. Effects of tourniquet during total knee arthroplasty: a prospective randomised study. J Bone Joint Surg Br. 1995;77:250-3.

11. Tai TW, Jou IM, Chang CW, Lai KA, Lin CJ, Yang CY. Nondrainage is better than 4-hour clamping drainage in total knee arthroplasty. Orthopedics. 2010;33.

12. Stucinskas J, Tarasevicius S, Cebatorius A, Robertsson O, Smailys A, Wingstrand H. Conventional drainage versus four hour clamping drainage after total knee arthroplasty in severe osteoarthritis: a prospective, randomised trial. Int Orthop. 2009;33:1275-8.

13. Tsumara N, Yoshiya S, Chin T, Shiba R, Kohso K, Doita M. A prospective comparison of clamping the drain or postoperative salvage of blood in reducing blood loss after total knee arthroplasty. J Bone Joint Surg Br. 2006;88:49-53. 